Original article

\title{
The association between melasma and iron profile: a case-control study
}

\author{
Azadeh Goodarzi, Elham Behrangi, Afsaneh Sadeghzadeh-Bazargan, Masoumeh Roohaninasab, \\ Fatemeh Sadat Hosseini-Baharanchi, Mahsa Shemshadi, Elham Vafaei
}

Iran University of Medical Sciences, Tehran, Iran

Received 9 June 2019, Revised 4 August 2019, Accepted 18 February 2020

(C) 2019, Goodarzi A., Behrangi E., Sadeghzadeh-Bazargan A., Roohaninasab M., Hosseini-Baharanchi F.S., Shemshadi M., Vafaei E. (C) 2019, Russian Open Medical Journal

Abstract: Background - Melasma is a chronic acquired localized hypermelanosis, causing aesthetic problem for women and impairing their quality of life. Evidence has suggested that hyperpigmentation can occur as a result of iron deficiency anemia and vitamin $B_{12}$ deficiency.

Aim - We aimed to evaluate the serum parameters of iron profile in melasma patients.

Material and Methods - This case-control study investigated 51 adult non pregnant women with melasma, compared with 51 controls, from patients referred to the Hospital, Tehran, Iran, 2017-2018. Melasma was diagnosed clinically according to the melasma area and severity index (MASI) score was calculated and recorded by the physician. The groups were compared in terms of vitamin $B_{12}$, folate, serum iron, hemoglobin $(\mathrm{Hb})$, mean corpuscular volume (MCV), total iron binding capacity (TIBC), and ferritin.

Results - The mean and standard deviation of the women's age was $36.89 \pm 8.88$ years, significantly higher in the group of patients with melasma. Comparing the serum parameters between the groups indicated no statistically significant difference in terms of mean levels of ferritin, $\mathrm{Hb}, \mathrm{MCV}$, iron, vitamin $\mathrm{B}_{12}, \mathrm{TIBC}$, and folate. The women in melasma group had a higher frequency in below normal range of ferritin and serum iron compared to the control group. None of the serum parameters were correlated with MASI.

Conclusion - The higher frequency of below normal range of ferritin and serum iron levels in women with melasma compared to the control group showed a possible association between these serum parameters and melasma.

Keywords: melasma, melanosis, ferritins, anemia, iron-deficiency, folate, vitamin $B_{12}$.

Cite as Goodarzi A, Behrangi E, Bazargan AS, Roohaninasab M, Hosseini-Baharanchi FS, Shemshadi M, Vafaei E. The association between melasma and iron profile: a case-control study. Russian Open Medical Journal 2020; 9: e0202.

Correspondence to Elham Vafaei. Address: Dermatology department, Rasoul Akram Hospital, Sattarkhan ST., Tehran, Iran. Phone: +98 9123882448. Email: elhamvafaeim@gmail.com.

\section{Introduction}

Melasma is a chronic acquired disease of the skin, caused by melanogenesis dysfunction [1], resulting in localized hypermelanosis [2]. Although medically considered a benign lesion, the aesthetic complication of melasma can impair women's quality of life and raise psychological concerns in the affected patients [3]. As to the evidence, melasma occurs in all races, ethnic populations, and geographic areas; however, the incidence of melasma in the generalpopulation is not clear and most studies describe the prevalence only according to the dermatologic visits [2, 4]. Generally, a higher prevalence is reported in populations with a darker skin phenotypes (Fitzpatrick III and IV), including Hispanic and Latin Americans, Asians, Middle Eastern and Africans [5] and a prevalence of about $40 \%$ is reported in Iranian women [6].

Although the exact pathogenesis of melasma is uncertain, a combination of genetic predisposition, in addition to environmental factors, like sun exposure, have been suggested to play a role in its higher incidence in summer on exposed parts of the body [4]. The higher incidence in women, especially during pregnancy, is also attributed to the role of female hormones on occurrence of melasma [7]. Hence, as melasma requires long-term treatment and the exact etiology is still unclear [8], research has focused on the underlying and attributing factors [9] in order to take a step towards a better understanding of the disease. In this regard, several serum parameters, like zinc, iron, copper, and magnesium, have been associated with skin lesions and melasma $[10,11]$. Vitamin $B_{12}$ deficiency is also associated with hyperpigmentation [12] and vitiligo [13]. However, its association with melasma is doubted [14].

Iron deficiency anemia is a public health problem with the highest prevalence in women of reproductive age [15] the same population susceptible for melasma. Evidence has suggested that hyper pigmentation can occur as a result of iron deficiency anemia and patients with melasma have been reported to have a lower serum levels of hemoglobin $(\mathrm{Hb})$, iron, ferritin and total iron binding capacity (TIBC) $[14,16,17]$. Hence, these studies had several limitations, such as limited sample size and not controlling the effect of confounders, like endocrine disorders and using medications like oral contraceptives, which could affect the results. Therefore, for a definite conclusion, we aimed to compare the serum levels of vitamin $\mathrm{B}_{12}$, folate, serum iron, $\mathrm{Hb}$, mean corpuscular volume (MCV), TIBC, and ferritin between nonpregnant women with and without melasma controlling for confounders. 
Table 1. Comparing serum measurements between melasma and control groups adjusted for age in linear regression

\begin{tabular}{|c|c|c|c|c|}
\hline Variant & Normal range, laboratory measurement method and device name & Melasma & Control & P-value \\
\hline Ferritin, $\mathrm{ng} / \mathrm{ml}$ & $10-90$, BT 3000 & $35.11 \pm 38.13$ & $36.73 \pm 24.68$ & 0.274 \\
\hline Hemoglobin, g/dl & 12-16, Full diff Sismex & $13.05 \pm 0.98$ & $13.07 \pm 0.79$ & 0.986 \\
\hline $\mathrm{MCV}, \mathrm{fl}$ & 77-97 femtoliter & $86.93 \pm 4.95$ & $84.88 \pm 5.61$ & 0.589 \\
\hline Serum iron, $\mathrm{micg} / \mathrm{dl}$ & 40-155, Full diff Sismex & $65.90 \pm 31.90$ & $61.72 \pm 24.39$ & 0.403 \\
\hline $\mathrm{TIBC}$, micg/dl & $230-440$, BT 3000 & $337.47 \pm 49.12$ & $316 \pm 49.44$ & 0.055 \\
\hline Vitamin $\mathrm{B}_{12}, \mathrm{pg} / \mathrm{ml}$ & 211-946, electrochemiluminescence, (ECL, made in Germany, 2010) & $341.45 \pm 212.56$ & $374 \pm 146.56$ & 0.491 \\
\hline Folate, $\mathrm{ng} / \mathrm{ml}$ & 4.6-34.8, electrochemiluminescence, (ECL, made in Germany, 2010) & $11.01 \pm 6.25$ & $13.13 \pm 6.04$ & 0.058 \\
\hline
\end{tabular}

Data presented as mean with standard deviation - M $\pm S D$

Table 2. Comparison the characteristics of the women in melasma and control group

\begin{tabular}{|c|c|c|c|c|}
\hline Characteristic & & Melasma & Control & P-value \\
\hline Age, year, $\mathrm{M} \pm \mathrm{SD}$ & - & $41.45 \pm 6.18$ & $32.33 \pm 8.86$ & $<0.001$ \\
\hline \multirow[t]{3}{*}{ Family history of melasma } & & & & 0.074 \\
\hline & Yes & $29(57)$ & 20 (39) & \\
\hline & No & $22(43)$ & $31(61)$ & \\
\hline \multirow[t]{3}{*}{ Using sunscreen, $\mathrm{n}(\%)$} & & & & 0.192 \\
\hline & Yes & $29(57)$ & $19(37)$ & \\
\hline & No & $22(43)$ & $32(63)$ & \\
\hline \multirow[t]{3}{*}{ Employment, $\mathrm{n}(\%)$} & & & & 0.685 \\
\hline & housewife & $32(63)$ & $30(59)$ & \\
\hline & Employed & $19(37)$ & $21(41)$ & \\
\hline \multirow[t]{3}{*}{ Marital status, $\mathrm{n}(\%)$} & & & & 0.308 \\
\hline & Married & $48(94)$ & 50 (98) & \\
\hline & Single & $3(6)$ & $1(1)$ & \\
\hline \multirow{4}{*}{ (hour), n (\%) } & & & & 0.295 \\
\hline & $<1$ & 10 (19) & $16(32)$ & \\
\hline & $1-2$ & $34(67)$ & $30(60)$ & \\
\hline & $>2$ & $7(14)$ & $4(8)$ & \\
\hline
\end{tabular}

$\mathrm{M} \pm \mathrm{SD}$ - data presented as mean with standard deviation; $\mathrm{n}(\%)$ - data presented as numbers and percentages.

\section{Material and Methods}

\section{Study sample}

The present case-control study investigated women with melasma who referred to the Dermatology Ward of the Rasoul Akram Hospital, Tehran, Iran, during 2017-2018.

The sample size was calculated at 51 in each group based on the mean serum iron level of the study by Qazi and colleagues [17], considering type I and II error of 0.05 and 0.20 , respectively. Accordingly, a total of 102 women were investigated in two groups: 51 patients in the case group and 51 patients in the control group. The inclusion criteria for both groups consisted of adult nonpregnant women aged 20 to 50 years old, with normal body mass index (BMI) (18.5-25 kg/m2), without autoimmune, thyroid or cardiac disease, pernicious anemia, celiac disease, Crohn's disease, leukemia, or cancer, and without history of hormone replacement therapy, alcohol use, smoking, oral contraceptive pills (OCP), phototoxic drugs, phenytoin, spironolactone, vitamin B12, and folic acid during last six months, who referred to the selected hospital's ward during the study period. Furthermore, the participants without vegetarian habit were included.

The participants of the case group were selected from patients with melasma by convenient sampling method from who referred to the Dermatology Ward of the hospital and participants of the control group were selected from patients who referred to other wards of the hospital, rather than dermatology, who were included by matching each control with each case. Patients were included into the study, after the researcher explained the study objectives and methods to the participants and asked them to read and sign the written informed consent, if they desired to participate in the study. They were ensured that all their information will be kept confidential and analyzed without revealing their personal information.

\section{Melasma diagnosis}

For the case group, all patients were visited by the clinic's physician and diagnosis of melasma was made clinically, by wood lamp, after ruling out other dermatologic/inflammatory disorders [18]. Also, themelasma area and severity index (MASI) score [19] was calculated by the physician and recorded in the study checklist.

\section{Variables}

For measurement of vitamin $\mathrm{B} 12$, folate, serum iron, $\mathrm{Hb}, \mathrm{MCV}$, TIBC, and ferritin, $15 \mathrm{cc}$ venous blood sample was taken from participants of both groups, kept in refrigerator, and sent to the hospital's laboratory immediately. Normal range of the serum parameters, the laboratory measurement techniques, and devices used are shown in Table 1.

\section{Statistical analysis}

Numbers with percentage and mean with standard deviation $(\mathrm{M} \pm \mathrm{SD})$ were used to describe qualitative and quantitative variables, respectively. Independent sample t-test was used to compare the quantitative variables with normal distribution. Kolmogorov-Smirnov test was used to investigate normality assumption. The comparison of the serum parameters between groups adjusted for significant covariates were done using multiple linear regression and logistic regression. Pearson's correlation coefficient was calculated to evaluate the association between the MASI scores and the serum parameters. The statistical software IBM SPSS Statistics for Windows, Version 22.0 (IBM Corp. Released 2013. Armonk, NY: IBM Corp), was used for statistical analysis. Pvalue less than 0.05 were considered significant.

\section{Results}

This study was completed by 51 women in the case group (with melasma) and 51 women without melasma (as the control group). The mean $\pm S D$ of the women's age was $36.89 \pm 8.88$ years. Table 2 shows that the characteristics of the women in melasma and control group were homogenous ( $P>0.05)$ except women's mean age which had a statistically significant difference between case and control groups ( $41.45 \pm 6.18$ vs. $32.33 \pm 8.86$, respectively) $(\mathrm{P}<0.001)$. Quantitative variables did not deviated from normality assumption $(\mathrm{P}>0.5)$. 
Table 3. Odds Ratio ( $95 \%$ confidence interval $(\mathrm{CI})$ ) for each serum parameter between the case and control groups adjusted for age

\begin{tabular}{|c|c|c|c|c|c|}
\hline $\begin{array}{l}\text { Serum } \\
\text { parameter }\end{array}$ & Categories & Melasma & Control & $\begin{array}{c}\text { Odds Ratio (95\% } \\
\text { CI) }\end{array}$ & $\begin{array}{c}P- \\
\text { value }\end{array}$ \\
\hline \multirow{3}{*}{$\begin{array}{c}\text { Ferritin, } \\
\mathrm{ng} / \mathrm{ml}\end{array}$} & $<10$ & $17(33)$ & $5(9.8)$ & $4.07(1.2,13.8)$ & 0.023 \\
\hline & $10-90^{*}$ & $29(56.9)$ & $44(86.3)$ & 1 & \\
\hline & $>90$ & $5(9.8)$ & $2(3.9)$ & $0.93(0.11,7.53)$ & 0.940 \\
\hline \multirow{2}{*}{$\begin{array}{l}\text { Hemoglobin, } \\
\mathrm{g} / \mathrm{dl}\end{array}$} & $<12$ & $5(9.8)$ & $5(9.8)$ & $1.22(0.27,5.63)$ & 0.791 \\
\hline & $>12$ & $46(90.2)$ & $46(90.2)$ & 1 & \\
\hline \multirow[t]{3}{*}{$\mathrm{MCV}, \mathrm{fl}$} & $<77$ & $1(2)$ & $3(5.9)$ & $0.73(0.06,8.5)$ & 0.800 \\
\hline & 77-97* & 49(96.1) & $48(94.1)$ & 1 & \\
\hline & $>97$ & $1(2)$ & $0(0)$ & - & \\
\hline \multirow{3}{*}{$\begin{array}{l}\text { Iron, } \\
\text { micg/dl }\end{array}$} & $<40$ & $11(21.6)$ & $5(9.8)$ & $3.99(1.04,15.25)$ & 0.042 \\
\hline & $40-155^{*}$ & $39(76.5)$ & $46(90.2)$ & 1 & \\
\hline & $>155$ & $1(2)$ & $0(0)$ & - & \\
\hline \multirow{3}{*}{$\begin{array}{l}\text { TIBC, } \\
\mathrm{micg} / \mathrm{dl}\end{array}$} & $<230$ & $1(2)$ & $2(3.9)$ & $1.4(0.09,21.8)$ & 0.814 \\
\hline & $230-440 *$ & $49(96.1)$ & $49(96.1)$ & 1 & \\
\hline & $>440$ & $1(2)$ & $0(0)$ & - & \\
\hline \multirow{3}{*}{$\begin{array}{l}\text { Vitamin } \mathrm{B}_{12} \\
\mathrm{pg} / \mathrm{ml}\end{array}$} & $<211$ & $13(25.5)$ & $5(9.8)$ & $3.47(0.95,12.6)$ & 0.058 \\
\hline & 211-946* & $36(70.6)$ & $46(90.2)$ & 1 & \\
\hline & $>946$ & $2(3.9)$ & $0(0)$ & - & \\
\hline \multirow{3}{*}{$\begin{array}{l}\text { Folate, } \\
\mathrm{ng} / \mathrm{ml}\end{array}$} & $<4.6$ & $4(7.8)$ & $1(2)$ & $5.7(0.51,63.1)$ & 0.158 \\
\hline & $4.6-34.8^{*}$ & $47(92.2)$ & $50(98)$ & 1 & \\
\hline & $>34.8$ & $0(0)$ & $0(0)$ & - & \\
\hline
\end{tabular}

* Normal range was considered as the reference group, $†$ Significant difference, in the case of zero frequency, the associated cell is omitted in the logistic regression modelling.

Table 4. The Pearson's correlation coefficient between the MASI score and the serum parameters

\begin{tabular}{lcc}
\hline Variant & Correlation & $P$-value \\
\hline Ferritin & -0.11 & 0.45 \\
Hemoglobin & -0.10 & 0.48 \\
MCV & 0.20 & 0.15 \\
Iron & 0.03 & 0.81 \\
TIBC & -0.15 & 0.29 \\
Vitamin $B_{12}$ & 0.16 & 0.24 \\
Folate & -0.03 & 0.83 \\
\hline
\end{tabular}

The patients' serum parameters are compared between the groups in Table 2. As indicated, there was no statistically significant difference between the groups in mean serum levels offerritin, $\mathrm{Hb}, \mathrm{MCV}$, iron, vitamin $\mathrm{B}_{12}, \mathrm{TIBC}$, and folate adjusted for female age $(P>0.05)$.

The serum parameters were categorized based on the normal range of each serum parameter and compared between the groups as shown in Table 3. It was showed that the odds of melasma for the female with below normal range of ferritin level $(<10)$ was $4.07(1.2,13.8)$ than the odds of melasma for the female with normal ferritin level $(P=0.023)$. The female with below normal range of iron level had the risk of melasma $3.99(1.04,15.25)$ than the female with normal iron level $(P=0.042)$. In addition, below normal range level of MCV decreased risk of female's melasma, however, it was not statistically significant (Table 3, $\mathrm{P}=0.814$ ). It was observed that the risk of melasma was higher in females with below normal range level of $H B(P=0.79)$, Vitamin $B 12(P=0.058)$, and folate $(P=0.158)$ in comparison to the females with that of in normal range. It was observed that the risk of melasma was lower in females with below normal range level of TIBC $(P=0.814)$ and $\operatorname{MCV}(P=0.8)$ in comparison to the females with that of in normal range.

Table 4 shows the assessment of the correlation between MASI and serum parameters in which none of the serum parameters were significantly associated with MASI ( $P>0.05)$.

\section{Discussion}

This study showed a higher frequency of below normal range ferritin level and serum iron levels in women with melasma, compared to the control group. Risk of melasma for the female with below normal range of ferritin level as well as iron was significantly higher than that of the patients with normal level which could imply the role of these serum parameters on melasma.

According to the evidence, hyperpigmentation is a symptom of vitamin $B_{12}$ deficiency, presenting prior to neurologic symptoms [20]. Vitamin $B_{12}$ is an important cofactor for methionine synthaseand its deficiency results in impaired methylation and DNA synthesis of haemoglobin and accumulation of homocysteine [21]. In melanogenesis, hyperpigmentation occurs due to depletion of intracellular glutathione, which inhibited tyrosinase activity [12]. Babaie and colleagues tested the serum level of vitamin $B_{12}$ in melasma and reported that only $1 / 70$ had a deficient vitamin $B_{12}$ level, while in the present study $13 / 51$ women $(25.5 \%)$ had a deficient vitamin $B_{12}$ level. This discrepancy between the results of the studies can be due to the different inclusion and exclusion criteria, as we excluded all patients who have used folic acid or vitamin $B_{12}$ supplementation, as well as different cut-off levels determined for vitamin $B_{12}$ deficiency. In addition, Babaie et al did not compare the results with a control group. In our study, comparing the frequency of vitamin $B_{12}$ deficiency between the case and controls revealed a nonsignificant association between melasma and vitamin $B_{12}$ deficiency. Although studies have determined the role of vitamin $B_{12}$ deficiency in different skin lesions and hyperpigmentation) [12, 13, 22], no other study has determined its deficiency in patients with melasma, and this study, as far as the authors are concerned, is the first to compare its level with a control group with nonsignificant association.

Moreover, it was found that the mean serum levels of $\mathrm{Hb}$, $M C V$, iron, vitamin $B_{12}, T I B C$, and folate were not different between the groups. Moreover, $\mathrm{Hb}, \mathrm{MCV}$, vitamin B12, TIBC, and folate these serum parameters were not associated with melasma risk.

TIBC represents the sum of all iron binding sites on transferrin, which tightly binds to the cell receptor and controls iron release into plasma; serum iron will be used by various cells and excess levels of iron will be stored as metabolically inactive form, ferritin [23]. TIBC is thus measured in diagnosis of iron deficiency anemia, iron overload, and chronic inflammatory disorders [24, 25]. The higher TIBC level in women with melasma showed lower iron storage, although the majority of the patients in both groups was within the normal range of TIBC. The lower iron storage of the patients with melasma was confirmed by a higher frequency of below normal range ferritin level. The results of the present study indicated that women with melasma have a higher frequency of anemia, and also have a lower iron storage. Also, the nutritional habits of patients can significantly affect the serum levels of these parameters, although we excluded vegetarians to minimize the confounding effect of nutrition on melasma and iron profile. Behrangi and colleagues compared the iron profile of 33 women with melasma with a control group and reported no significant difference between the groups in serum iron, ferritin or TIBC [16]. Their results confirmed that of the present study on no difference between the patients with melasma and the control group in terms of TIBC, but were inconsistent with the results of the present study in terms of serum ferritin and serum iron levels. This 
discrepancy could be due to the difference of the baseline serum level of ferritin and serum iron in the study population. In the present study, we tried to exclude any patients with chronic diseases or supplementation use to control the confounders affecting TIBC, serum iron, and ferritin levels. Babaie et al reported low $\mathrm{Hb}$ and serum iron levels in $14.3 \%$ and $8.6 \%$ of women with melasma [14], while in the present study, $9.8 \%$ of patients with melasma had low $\mathrm{Hb}$ level and $21.6 \%$ had low serum iron level. This difference can be attributed to the different frequency of anemia in different populations and may also differ according to the underlying diseases and different age groups of the studied population [15]. In the present study, the mean age of the groups had significant differences, which could also be attributed to different iron profile, so we adjusted the analysis regarding the age difference. The study by Qazi et al (2017), comparing 70 nonpregnant women with melasma and the control group, showed a lower mean serum iron and ferritin levels, beside a higher TIBC in the case group [17]. These results showed a higher frequency of anemia and lower iron storage in women with melasma, which was consistent to the results of the present study, although the $\mathrm{Hb}$ and TIBC levels did not differ between the groups in our study and lower iron storage, was indicated by the lower mean serum iron and higher frequency of below normal range ferritin level. These results could indicate that women with melasma had low iron levels and storage, but as far as evident in thevitamin $B_{12}$ and folate although mean levels were lower in melasma group but they were not expressive enough, which could be followed by future studies.

In the present study, all variables were tested for association with MASI, but the results showed no significance. On the contrary to these results, Qazi et al stated a positive correlation between MASI score and iron profile [17], which could be also due to the lower mean serum iron in the study by Qazi et al, while only reduced iron storage was detected in the patients of the present study.

Anemia is a prevalent health problem, especially in women of reproductive age [15], who are the main target population of melasma, a disturbing skin disease in women, impairing patients' quality of life [3]. The target population of the highest frequency of anemia and melasma are the same. Furthermore, the frequency of melasma seems to be higher in pregnant women due to the increased levels of placental, ovarian and pituitary hormones, which results in increased transcription of tyrosinase and dopachrome tautomerase and pigmentation during pregnancy [2, 26]. The same population, pregnant women, have a high frequency of anemia [27]. These two, the higher frequency of anemia and melasma during pregnancy, may be associated with each other, which have to be further investigated. In the present study, we did not include pregnant women, due to the complex situations and hormone secretion in pregnancy, which may affect this association.

The present study could successfully compare all these parameters between the case and control groups, while the one study investigating all these parameters had the major limitation of a small sample size. Like any other study, our study could also have several limitations. First, the participants did not match with each other in terms of demographics. Second, all participants were selected from one center that reduce the generalizability of the results. Furthermore, the case-control nature of the study limited confirming causal relationships, although there was no statistically significant association between the variables. Meanwhile, as the first valid study to investigate this association in an Iranian population, the present study could provide valuable information for the researchers and clinicians.

\section{Conclusion}

The results of the present study showed that the iron profile of women with melasma is different from that of controls. The higher frequency of below normal range ferritin and serum iron levels in the case group showed the possible role of anemia in melasma.

\section{Limitations}

Although lower frequency of above normal range vitamin $B_{12}$ and folate showed a lower iron storage in women with melasma, the initial hypothesis of the study (the association of melasma as hyperpigmentation with vitamin $B_{12}$ and serum folate deficiency) was not confirmed, as hemoglobin and MCV levels were not different between the groups. Although we excluded possible factors that could affect vitamin $B_{12}$ and serum folate levels and pathogenesis of hyperpigmentation and melasma, there could be several factors affecting the two multifactorial diseases, vitamin $B_{12}$ deficiency and melasma, which could not be controlled in the present study. Future randomized clinical trial or cohort studies can shed light on the association between serum folate and vitamin $\mathrm{B}_{12}$ and melasma.

\section{Ethical approval}

The study was approved by the Ethics Committee of Iran University of Medical Sciences (code: IR.IUMS.FMD.REC1395.08921215097), and has been performed in accordance with the ethical standards as laid down in the 1964 Declaration of Helsinki and its later amendments or comparable ethical standards. Informed consent was given to all participants.

\section{Acknowledgement}

The authors would like to thank the Rasoul Akram Hospital Clinical Research Development Center (RCRDC) for its technical and editorial assists.

\section{Conflict of interest}

The authors declare that they have no conflict of interest.

\section{References}

1. Kang HY, Suzuki I, Lee DJ, Ha J, Reiniche P, Aubert J, et al. Transcriptional profiling shows altered expression of wnt pathway- and lipid metabolism-related genes as well as melanogenesis-related genes in melasma. J Invest Dermatol 2011; 131(8): 1692-1700. https://doi.org/10.1038/iid.2011.109.

2. Handel AC, Miot LD, Miot HA. Melasma: a clinical and epidemiological review. An Bras Dermatol 2014; 89(5): 771-782. https://doi.org/10.1590/abd1806-4841.20143063.

3. Katsambas A, Soura E. Quality of life in melasma. In: Melasma and vitiligo in brown skin. E. Handog, M. Enriquez-Macarayo, eds. New Delhi: Springer, 2017: 169-175. https://doi.org/10.1007/978-81-3223664-1 18.

4. Bagherani N, Gianfaldoni S, Smoller B. An overview on melasma. Pigmentary Disorders 2015; 2(10): 218. https://doi.org/10.4172/23760427.1000216.

5. Khoza N, Dlova N, Mosam A. Epidemiology and global distribution of melasma. In: Melasma: a monograph 1st ed. New Delhi: Jaypee Brothers Medical Publisher, 2015: 1-3. https://doi.org/10.5005/ip/books/12401 2. 
6. Edalatkhah $\mathrm{H}$, Amani $\mathrm{F}$, Rezaifar $\mathrm{G}$. Prevalence of melasma in women in Ardebil city in 2002. Iran J Dermatol 2004; 7(2): 72-77. https://www.sid.ir/en/journal/ViewPaper.aspx?ID=48038.

7. Mahmood K, Nadeem M, Aman S, Hameed A, Kazmi AH. Role of estrogen, progesterone and prolactin in the etiopathogenesis of melasma in females. J Pak Assoc Dermatol 2016; 21(4): 241-247. http://www.jpad.com.pk/index.php/jpad/article/view/483.

8. Lajevardi V, Ghayoumi A, Abedini R, Hosseini H, Goodarzi A, Akbari Z, et al. Comparison of the therapeutic efficacy and safety of combined oral tranexamic acid and topical hydroquinone $4 \%$ treatment vs. topical hydroquinone $4 \%$ alone in melasma: a parallel-group, assessorand analyst-blinded, randomized controlled trial with a short-term follow-up. J Cosmet Dermatol 2017; 16(2): 235-242. https://doi.org/10.1111/jocd.12291.

9. Amin N, Mashhood AA, Bilal A. Association of epidermal melasma with skin phenotypes and other contributing factors. J Pak Assoc Dermatol 2016; 26(3): 188-192. https://pdfs.semanticscholar.org/7197/6346aaa4dbcb9a41f6bb81031 99f18e7b022.pdf

10. Rostami Mogaddam M, Safavi Ardabili N, Iranparvar Alamdari M, Maleki N, Aghabalaei Danesh M. Evaluation of the serum zinc level in adult patients with melasma: Is there a relationship with serum zinc deficiency and melasma? J Cosmet Dermatol 2018; 17(3): 417-422. https://doi.org/10.1111/jocd.12392.

11. Ping W, Guohua L, Huihui L, Zhengwen W. Determination of trace elements in pregnant and non-pregnant patients with melasma. Trace Elem Sci 1997; 8. http://en.cnki.com.cn/Article en/CJFDTotalGWYS199708006.htm.

12. Niiyama $S$, Mukai $H$. Reversible cutaneous hyperpigmentation and nails with white hair due to vitamin B 12 deficiency. Eur J Dermatol 2007; 17(6): 551-552. https://doi.org/10.1684/ejd.2007.0285.

13. Karadag AS, Tutal E, Ertugrul DT, Akin KO, Bilgili SG. Serum holotranscobalamine, vitamin $B_{12}$, folic acid and homocysteine levels in patients with vitiligo. Clin Exp Dermatol 2012; 37(1): 62-64. https://doi.org/10.1111/j.1365-2230.2011.04142.x.

14. Najad SB, Khodaiiani E, Herizchi H, Mehrabi $P$. Frequency of iron deficiency anemia, folate and vitamin $\mathrm{B}_{12}$ deficiency in patients with melasma. Med J Tabriz Univ Med Sci 2012; 34(2): 12-15. https://www.sid.ir/en/journal/ViewPaper.aspx?id=284349.

15. WHO. The global prevalence of anaemia in 2011. Geneve: World Health Organization, 2015; 43 p. https://apps.who.int/iris/handle/10665/177094.

16. Behrangi E, Baniasadi F, Esmaeeli S, Hedayat K, Goodarzi A, Azizian Z. Serum iron level, ferritin and total iron binding capacity level among nonpregnant women with and without melasma. J Res Med Sci 2015; 20(3): 281-283. https://www.ncbi.nlm.nih.gov/pubmed/26109976.

17. Qazi I, Dogra NK, Dogra D. Serum Iron profile in Female patients of Melasma: A case control study. Asian Pac J Health Sci 2017; 4 (2): 141146. https://doi.org/10.21276/apjhs.2017.4.2.24.

18. Dermatology atlas for skin of color. D. Jackson-Richards, A.G. Pandya, eds. Berlin: Springer-Verlag Berlin Heidelberg, 2014; 321 p. https://doi.org/10.1007/978-3-642-54446-0.

19. Pandya AG, Hynan LS, Bhore R, Riley FC, Guevara IL, Grimes P, et al. Reliability assessment and validation of the melasma area and severity index (MASI) and a new modified MASI scoring method. J Am Acad Dermatol 2011; 64(1): 78-83. https://doi.org/10.1016/j.jaad.2009.10.051.

20. Stabler SP. Clinical practice. Vitamin B12 deficiency. N Engl J Med 2013; 368(2): 149-160. https://doi.org/10.1056/NEJMcp1113996.

21. Kräutler B. Vitamin B12: chemistry and biochemistry. Biochem Soc Trans 2005; 33(Pt 4):806-810. https://doi.org/10.1042/BST0330806.

22. Kannan R, Ng MJ. Cutaneous lesions and vitamin B12 deficiency: an often-forgotten link. Can Fam Physician 2008; 54(4): 529-532. https://www.ncbi.nlm.nih.gov/pubmed/18413300.
23. Soldin OP, Bierbower LH, Choi JJ, Choi JJ, Thompson-Hoffman S, Soldin SJ. Serum iron, ferritin, transferrin, total iron binding capacity, hs-CRP, LDL cholesterol and magnesium in children; new reference intervals using the Dade Dimension Clinical Chemistry System. Clin Chim Acto 2004; 342(1-2): 211-217. https://doi.org/10.1016/j.cccn.2004.01.002.

24. Gottschalk R, Wigand R, Dietrich CF, Oremek G, Liebisch F, Hoelzer D et al. Total iron-binding capacity and serum transferrin determination under the influence of several clinical conditions. Clin Chim Acta 2000; 293(1-2): 127-138. https://doi.org/10.1016/s0009-8981(99)00242-9.

25. Taher AT, Musallam KM, Inati A. Iron overload: consequences, assessment, and monitoring. Hemoglobin 2009; 33 Suppl 1: S46-S57. https://doi.org/10.3109/03630260903346676.

26. Moin A, Jabery Z, Fallah N. Prevalence and awareness of melasma during pregnancy. Int J Dermatol 2006; 45(3): 285-288. https://doi.org/10.1111/i.1365-4632.2004.02470.x

27. Esmat B, Mohammad R, Behnam S, Shahrzad M, Soodabeh T, Minoo A et al. Prevalence of iron deficiency anemia among Iranian pregnant women; a systematic review and meta-analysis. J Reprod Infertil 2010; 11(1): 17-24. https://www.ncbi.nlm.nih.gov/pubmed/23926476.

\section{Authors}

Azadeh Goodarzi - MD, Assistant Professor, Department of Dermatology, Rasoul Akram Hospital, Iran University of Medical Sciences, Tehran, Iran. https://orcid.org/0000-0002-1249-4429.

Elham Behrangi - MD, Associate Professor, Department of Dermatology, Rasoul Akram Hospital, Iran University of Medical Sciences, Tehran, Iran. https://orcid.org/0000-0002-6545-3460.

Afsaneh Sadeghzadeh Bazargan - MD, Assistant Professor, Department of Dermatology, Rasoul Akram Hospital, Iran University of Medical Sciences, Tehran, Iran. https://orcid.org/0000-0003-1102-6241.

Masoomeh Rohaninasab - MD, Assistant Professor, Department of Dermatology, Rasoul Akram Hospital, Iran University of Medical Sciences, Tehran, Iran. https://orcid.org/0000-0002-2862-6422.

Fatemeh Sadat Hosseini-Baharanchi - MA, MS, PhD, Assistant Professor, Minimally Invasive Surgery Research Center, \& Department of Biostatistics, School of Public Health, Iran University of Medical Sciences, Tehran, Iran. https://orcid.org/0000-0003-1633-2635/

Mahsa Shemshadi - MD, Resident of Dermatology, Rasoul Akram Hospital, Iran University of Medical Sciences, Tehran, Iran. https://orcid.org/00000002-6912-0532.

Elham Vafaei - MD, General Practitioner, Rasoul Akram Hospital, Iran University of Medical Sciences, Tehran, Iran. https://orcid.org/0000-0002$\underline{5684-6625}$. 